\title{
Prognostic significance of neutrophil-to- lymphocyte ratio in rectal cancer: a meta-analysis
}

This article was published in the following Dove Press journal:

OncoTargets and Therapy

25 May 2016

Number of times this article has been viewed

\section{Yi-wei Dongl,* \\ Yan-qiang $\mathrm{Shi}^{1, *}$ \\ Li-wen $\mathrm{He}^{1, *}$ \\ Pei-zhu Su ${ }^{2}$}

'The Second Clinical Medical School, Southern Medical University, 2Department of Gastroenterology, Zhujiang Hospital, Southern Medical University, Guangzhou City, Guangdong Province, People's Republic of China

*These authors contributed equally to this work
Correspondence: Pei-zhu Su Department of Gastroenterology, Zhujiang Hospital, Southern Medical University, 253 Industrial Avenue, Haizhu District, 5I0280, Guangzhou City, Guangdong Province, People's Republic of China

Tel +8620I55 21287895

Email supeizhu1986@163.com
Background: Inflammatory responses play decisive roles in tumor development, immune surveillance, and responses to therapy. High neutrophil-to-lymphocyte ratio (NLR), as an inflammation index, has been reported to be a predictor for poor prognosis of various cancers. The purpose of this meta-analysis was to evaluate the prognostic value of NLR in patients with rectal cancer.

Methods: A comprehensive search of the literature was conducted through PubMed and EMBASE. Pooled hazard ratio (HR) with $95 \%$ confidence interval (CI) was used to evaluate the association between NLR and three outcomes: overall survival, disease-free survival, and recurrence-free survival.

Results: Seven cohorts involving 959 patients were included in this meta-analysis. Our pooled results demonstrated that elevated NLR was associated with poor overall survival (HR: 13.41, 95\% CI: 4.90-36.72), disease-free survival (HR: 4.37, 95\% CI: 2.33-8.19), and recurrence-free survival (HR: $3.64,95 \%$ CI: 1.88-7.05).

Conclusion: An elevated NLR is a valuable and easily available prognostic marker for rectal cancer. It is associated with unfavorable overall survival, disease-free survival, and recurrencefree survival. NLR could be a useful candidate factor for making treatment decisions for individual patients with rectal cancer.

Keywords: neutrophil-to-lymphocyte ratio, rectal cancer, prognosis, meta-analysis, overall survival, disease-free survival

\section{Introduction}

The morbidity and mortality of rectal cancer are high in the Western world although there is a significant improvement in diverse treatment methods and overall survival (OS) in the last decade. ${ }^{1}$ The Tumor Node Metastasis (TNM) staging system and Dukes' staging system are beneficial to stratify patients into different treatment strategies, but the prognosis varies even in patients at the same stage. Prognostic significance of supplementary items to current tumor staging system has been investigated in recent years, such as inflammatory factors, proteins, and genes; nevertheless, no reliable markers have been found. ${ }^{2}$ Therefore, the introduction of some reliable and affordable markers is essential for predicting prognosis for patients with rectal cancer and providing personalized therapy strategies.

The relationship between cancer and inflammation was first reported by Virchow in $1863 .{ }^{3}$ In recent years, an increasing number of studies have reported the association between systematic inflammatory response and prognosis of cancer. ${ }^{4}$ An elevated peripheral blood neutrophil-to-lymphocyte ratio (NLR), a serum-based inflammatory index, has been recognized as a predictor of poor prognosis in various cancers, including breast cancer, renal cell carcinoma, gastric cancer, and hepatocellular carcinoma. ${ }^{5-8}$ Accumulating evidence indicated the potential relationship between elevated NLR (c) $\mathrm{NC}$ and incorporate the Creative Commons Attribution - Non Commercial (unported, v3.0) License (http:///reativecommons.org/licenses/by-nc/3.0/). By accessing the work you
hereby accept the Terms. Non-commercial uses of the work are permitted without any further permission from Dove Medical Press Limited, provided the work is properly attributed. For permission for commercial use of this work, please see paragraphs 4.2 and 5 of our Terms (https://www.dovepress.com/terms.php). 
and poorer survival in rectal cancer. But these results remain inconclusive due to the different study designs and limited number of patients across studies. ${ }^{9,10}$ Therefore, we systematically performed a meta-analysis based on relevant studies to explore the prognostic value of NLR on survival in patients with rectal cancer.

\section{Materials and methods} Literature research

PubMed and EMBASE were systematically searched up with search strategy based on the following terms: ("neutrophil lymphocyte ratio" OR "neutrophil-to-lymphocyte ratio" OR "neutrophil-lymphocyte ratio" OR NLR) AND ("rectal neoplasms" OR "rectal neoplasm*" OR "rectal tumor*” OR "rectal cancer*" OR "rectum tumor*" OR "rectum cancer*" OR "rectum neoplasm*"). Any restriction including language, study design, human research, and so on is not permitted. And the latest search was conducted on November 11, 2015. Besides, manual search of references in relevant reviews was performed to receive potential articles.

\section{Inclusion and exclusion criteria}

Two authors independently reviewed the candidate studies, and the discrepancies were resolved by discussions. The guidance of selecting eligible studies for our meta-analysis was accorded to predefined inclusion and exclusion criteria. Inclusion criteria were 1) the diagnosis of rectal cancer confirmed by pathological examination; 2) the associations between NLR and OS, disease-free survival (DFS) or recurrence-free survival (RFS); 3) sufficient data to estimate the hazard ratio (HR) and 95\% confidence interval (CI); and 4) the NLR measured before treatment. Exclusion criteria were as follows: 1) study types including case report, case series, letter, editorial, and review; 2) insufficient or unspecific data of NLR; 3) languages other than English; and 4) nonhuman researches.

\section{Data extraction and quality assessment}

Data of identified studies were extracted by two investigators independently. The latest study with complete data was included in the meta-analysis when the data overlapped cross studies. The following information was extracted: author, year of publication, country of study, sample size, age, TNM stage, treatment, cutoff value, survival analysis, duration, and follow-up time. The quality assessment of included studies was conducted by two reviewers independently using the Newcastle-Ottawa Quality Assessment Scale. ${ }^{11}$ Any discrepancies were discussed and resolved in the process.

\section{Statistical analysis}

HR and 95\% CI were gained directly from included studies or estimation based on methods by Parmar et al. ${ }^{12} \mathrm{We}$ estimated pooled HRs on prognosis (OS, DFS, and RFS) to evaluate the predictive value of NLR. The significance of the pooled HRs was determined by $Z$-test, and the level of statistical significance was established as $P<0.05$. The heterogeneity among studies was checked by the $Q$-test and Higgins $I^{2}$ statistic. ${ }^{13,14}$ If the $P$-value for the heterogeneity test was $>0.05$, the Mantel-Haenszel method-based fixed effects model was used to calculate the pooled HR. ${ }^{15}$ Otherwise, the DerSimonian and Laird ${ }^{16}$ method-based random effects model was used. Potential publication bias was evaluated by visual inspection of the Begg's funnel plots, and we also performed an Egger's linear regression test $\left(P<0.05\right.$ was considered a significant publication bias) ${ }^{17}$ All the statistical analyses were performed using a software program, STATA Version 12.0 (StataCorp LP, College Station, TX, USA).

\section{Results}

\section{Study selection and characteristics}

The flowchart of the included studies is shown in Figure 1. A total of 242 records were identified through database search and other sources in the initial search. According to the predefined inclusion and exclusion criteria, seven studies with 959 patients published from 2010 to 2015 were included in the meta-analysis. Main characteristics of the included studies are listed in Table 1. The countries of studies included Japan $(n=2)$, the UK $(n=2)$, People's Republic of China $(n=2)$, and Korea $(n=1)$. As for the quality assessment, the scores of included trials ranged from 4 to 7 .

\section{NLR and OS in rectal cancer}

A total of six trials comprising 857 patients reported HR for OS. ${ }^{2,9,10,18-20}$ A pooled HR of 13.408 (95\% CI: 4.896-36.715, Figure 2) showed that a higher NLR was expected to an unfavorable OS. The results of heterogeneity test indicated that there was no significant heterogeneity $\left(P=0.852, I^{2}=0.0 \%\right.$, Figure 2). Due to relatively minor heterogeneity, further meta-regression or subgroup analysis was not conducted.

\section{NLR and DFS in rectal cancer}

A total of three trials including 403 patients reported HR for DFS. ${ }^{2,10,18}$ An elevated NLR was associated with a HR for DFS of 4.368 (95\% CI: 2.329-8.190, Figure 3) with low heterogeneity $\left(P=0.411, I^{2}=0.0 \%\right.$, Figure 3$)$. 


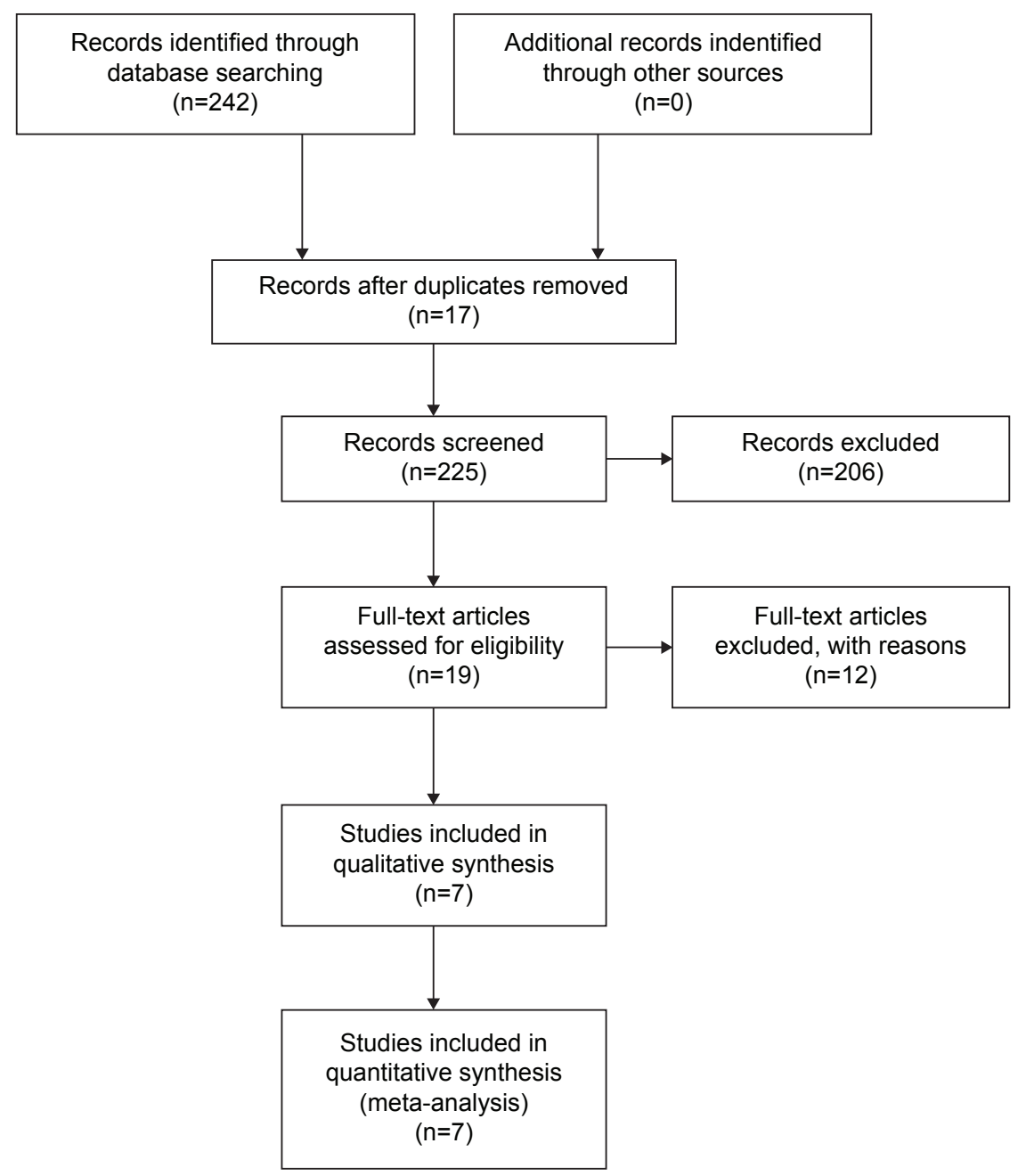

Figure I Flowchart of the included studies.

\section{NLR and RFS in rectal cancer}

A total of three trials comprising 433 patients reported HR for RFS. ${ }^{9,20,21}$ An elevated NLR was related to a HR for RFS of 3.636 (95\% CI: 1.876-7.048, Figure 4) with low heterogeneity $\left(P=0.482, I^{2}=0.0 \%\right.$, Figure 4$)$.

\section{Sensitivity analysis and publication bias}

Sensitivity analysis was performed by excluding one individual study each time to assess the influence of each individual study on the pooled HRs for OS. The corresponding HRs did not change considerably, suggesting that the findings of this meta-analysis are credible.

Begg's funnel plot and Egger's test were conducted to evaluate the publication bias of the included cohorts in OS. Funnel plot shape did not reveal any obvious evidence of asymmetry. The $P$-value for Egger's test in the HR for OS was 0.057 (Figure 5). Overall, the above results suggested that publication bias was not evident in this meta-analysis.

\section{Discussion}

Prior studies have suggested the association between high NLR and poor outcome of patients with various types of cancer. Our analysis combined the outcomes of 959 patients with rectal cancer from seven studies to evaluate the prognostic effect of NLR, demonstrating that the elevated NLR was associated with unfavorable OS (HR: 13.408), DFS (HR: 4.368), and RFS (HR: 3.636). The results of sensitivity analyses suggested that our review was reliable and stable. There was no significant publication bias in our study. To our knowledge, the present study is the first meta-analysis assessing the association between NLR and prognosis in rectal cancer.

The mechanism underlying the association between elevated NLR and poor outcome in rectal cancer remains complicated. The first potential mechanism is that inflammation contributes to the construction of "tumor microenvironment" by supplying tumor-promoting cytokines that active some 


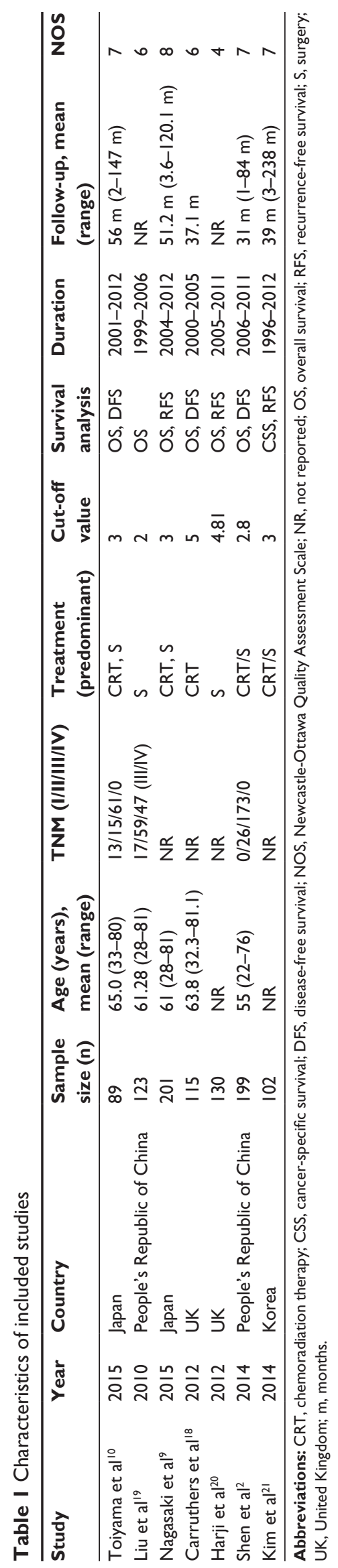

signalings, such as nuclear factor $\kappa \mathrm{B}(\mathrm{NF}-\kappa \mathrm{B})$, transducer and activator of transcription 3 (STAT3). ${ }^{22,23}$ These transcription factors possibly induce genes in premalignant cells to stimulate cell proliferation and survival, as well as angiogenesis, invasiveness, motility, chemokine, and cytokine production. ${ }^{24}$ Particularly, interleukin 6 (IL-6) is an important tumor-promoting cytokine and its tumor-promoting effect is mainly exerted via STAT3 ${ }^{25}$ Serum concentration of IL-6 has been reported to be increased in colorectal cancer and other 12 different cancer types and has been associated with tumor stage and adverse prognosis. ${ }^{26}$ There are also other tumor-promoting cytokines such as IL-23, tumor necrosis factor- $\alpha$, and IL-1 $\beta \cdot{ }^{27-30}$ Second, lymphocytes can reduce tumor infiltration via a series of subtypes, lymphocytes, activated T-cells, and natural killer cells, which has been shown to improve the survival of patients with cancer. ${ }^{31-33}$ The dysfunction of T-lymphocyte, as one common kind of tumor infiltrating lymphocyte, could result in immune escape of tumor cells. ${ }^{34,35}$ The lymphocyte-dependent antitumoral immune response could be inhibited by the recruitment of inflammatory cells. In addition, inflammation promotes the accumulation of myeloid-derived suppressor cells and regulatory T-cells (Tregs), both of which downregulate the immune surveillance and antitumor immunity. ${ }^{36,37}$ In general, elevated NLR caused by neutrophilia or lymphopenia, denotes enhanced inflammatory response and suppression of immunity. As tumorigenesis and tumor progression in humans often take decades, NLR as a predictor can be used to tailor the personalized treatment strategy.

In addition to NLR, other markers of inflammatory response, such as platelet lymphocyte ratio (PLR) and the modified Glasgow prognostic score (mGPS), have been shown to be prognostic factors for survival in colorectal cancer. ${ }^{38,39}$ The mGPS was based on C-reactive protein and albumin. Toiyama et a ${ }^{40}$ reported that elevated C-reactive protein was an adverse prognostic factor for OS and DFS in 84 patients with locally advanced rectal cancer in 2013. Roxburgh et a ${ }^{41}$ reported that mGPS is an important independent predictor of 3 -year survival in colon and rectal cancers. The multivariate analysis of Powell showed that mGPS $(P=0.028)$ was independently related to cancer-specific survival. ${ }^{42}$ None of our included studies have reported the relationship between mGPS and survival in the multivariate analysis. However, these parameters are not routinely measured in daily clinical practice. In contrast, NLR owns the advantage of low economic cost and wide availability over the above laboratory items. NLR can be obtained from peripheral blood routine test, which is routinely exanimated before the treatment and 


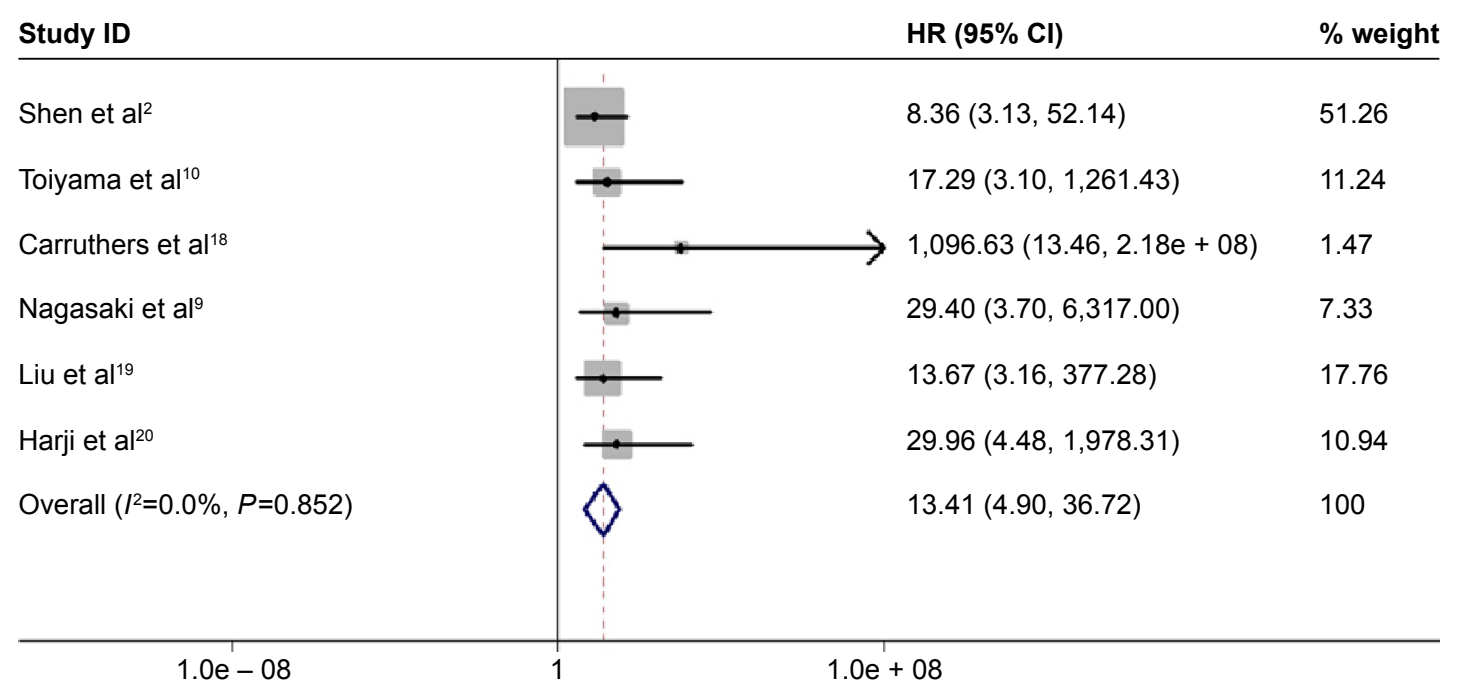

Figure 2 Forest plots of studies evaluating HR with $95 \% \mathrm{Cl}$ of NLR for OS.

Abbreviations: $\mathrm{Cl}$, confidence interval; $\mathrm{HR}$, hazard ratio; NLR, neutrophil-to-lymphocyte ratio; OS, overall survival.

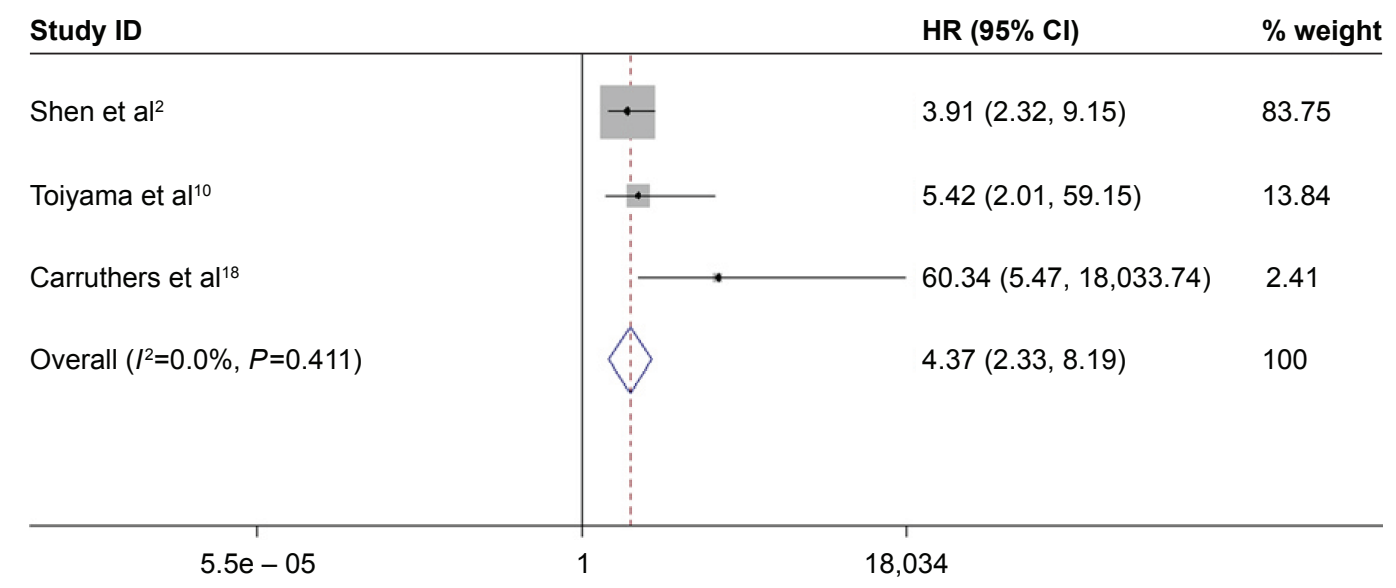

Figure 3 Forest plots of studies evaluating HR with $95 \% \mathrm{Cl}$ of NLR for DFS.

Abbreviations: $\mathrm{Cl}$, confidence interval; DFS, disease-free survival; HR, hazard ratio; NLR, neutrophil-to-lymphocyte ratio.

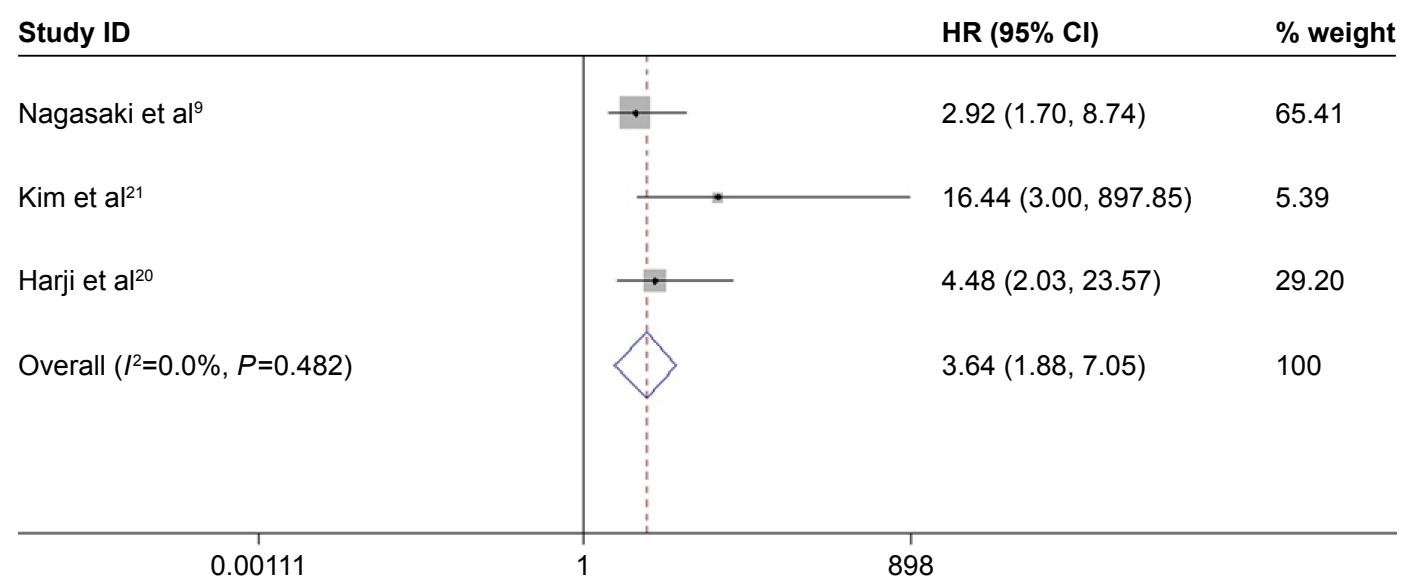

Figure 4 Forest plots of studies evaluating HR with $95 \% \mathrm{Cl}$ of NLR for RFS.

Abbreviations: $\mathrm{Cl}$, confidence interval; HR, hazard ratio; NLR, neutrophil-to-lymphocyte ratio; RFS, recurrence-free survival. 


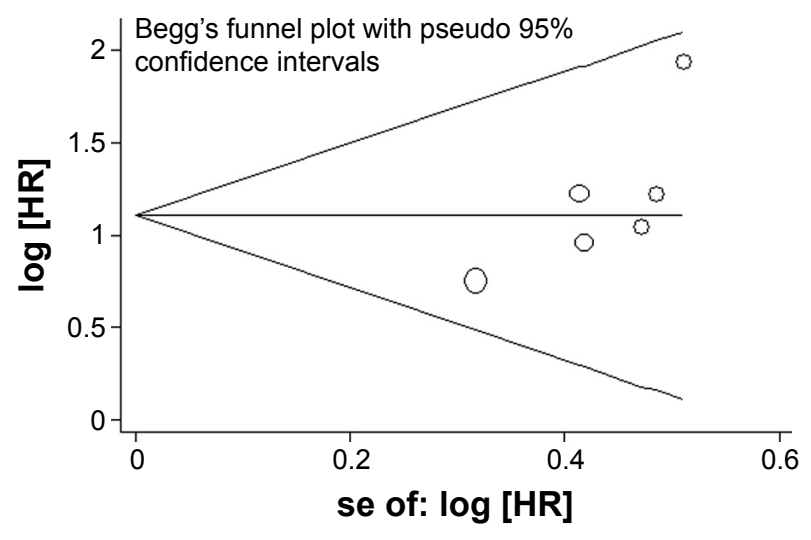

Figure 5 Begg's funnel plot for the assessment of potential publication bias. Abbreviations: HR, hazard ratio; se, standard error.

consequently costs no extra expenditure. Changes of NLR are easily monitored during the process of treatment. PLR is available from a routine blood test such as NLR. In a study of 200 patients undergoing curative resection for rectal cancer, both NLR and PLR were shown to be prognostic biomarkers of OS. ${ }^{43}$ Two of our included studies reported the information about PLR. In the study of Toiyama we included, high platelet before chemoradiation therapy is an independent predictor of poor OS and DFS in rectal cancer patients, while platelet and NLR were significantly correlated. ${ }^{10}$ The study of Carruthers reported that higher PLR showed no significant association with OS, DFS, and time to local recurrence, while higher NLR was shown to be associated with all outcomes. ${ }^{18}$ Based on the limited studies providing comparison between NLR and other inflammatory markers, we think that it is premature to conclude which inflammatory marker is better, and more relevant studies are needed to draw a conclusion.

However, NLR is a parameter in low specificity. An elevated NLR could be induced by many concurrent diseases and conditions, such as infections and medications. NLR also appears to be prognostic in noncancer conditions such as acute pancreatitis or cardiac events. ${ }^{44,45}$ Some of our included studies did not control the different concurrent conditions or report them in detail. We consider these as confounding factors for the explanation of the prognostic role of NLR.

Here are some limitations in this study. First, only summarized data rather than individual data were available for analysis. Second, the number of studies we included is small. We included one abstract with poor quality in order to get more information. Third, we only included studies reporting HR for OS, DFS, or RFS and consequently other studies focusing on the prognostic effect of NLR were excluded because of their different effect sizes or endpoints. Fourth, among the including studies, the only abstract reported unclear endpoint of multivariate HR, thus we selected the univariate HR. Because of large variations in the covariates of multivariate HR across included studies, sensitivity analyses in accordance with adjustment variables were not conducted. Both of the above may lead to bias toward overestimation of prognostic effect of NLR. Fifth, the unified cutoff value for high NLR contributes to between-studies heterogeneity. Sixth, there are some other powerful variables associated with survival of rectal cancer, such as level of serum alpha fetoprotein, tumor location, tumor stage, and depth of invasion. The correlations between NLR and the above factors remain unclear. There was no sufficient data for us to perform further subgroup analysis. Seventh, other existing inflammatory conditions were not discussed in the original studies and the control samples with inflammation from noncancer patients were lacking in the included studies.

In our opinion, with regard to research design, the strategies to bring NLR into a clinically relevant biomarker are first, the investigators could include control samples with inflammation from noncancer patients to test the specificity of NLR. Second, researchers should perform multivariate analysis to gain a HR for NLR's prognostic role and take many clinicopathological parameters into account, such as tumor stage before chemoradiation therapy, tumor size, tumor location, adjuvant chemotherapy, the prechemoradiation therapy serum carcinoembryonic antigen level, alpha fetoprotein, ypTNM staging, depth of invasion, histological type, pathological tumor response, lymph node metastasis, and so on. The multivariate HR of the specific variable removes the influences of other variables and assesses the prognostic effect of NLR as an independent factor.

\section{Conclusion}

Our results indicated that elevated NLR might be a poor prognostic factor for survival in patients with rectal cancer. NLR, an inexpensive, widely available, and reproducible index, is a promising prognostic indicator for patients with rectal cancer. It could be useful in stratifying patients and determining individual therapy plans. However, these results need to be interpreted cautiously before the clinical application of NLR as an independent biomarker because of the limitations listed above. More multicenter prospective cohorts and large-scale investigations are warranted to validate the value of NLR in the prognosis of rectal cancer, thus leading to more detailed patient selection when applying it to clinical decisions.

\section{Disclosure}

Yi-wei Dong, Yan-qiang Shi, and Li-wen He are joint first authors for this study.

The authors report no conflicts of interest in this work. 


\section{References}

1. Nielsen LB, Wille-Jorgensen P. National and international guidelines for rectal cancer. Colorectal Dis. 2014;16(11):854-865.

2. Shen L, Zhang H, Liang L, et al. Baseline neutrophil-lymphocyte ratio $(>/=2.8)$ as a prognostic factor for patients with locally advanced rectal cancer undergoing neoadjuvant chemoradiation. Radiat Oncol. 2014;9:295.

3. Balkwill F, Mantovani A. Inflammation and cancer: back to Virchow? Lancet. 2001;357(9255):539-545.

4. Mantovani A, Allavena P, Sica A, Balkwill F. Cancer-related inflammation. Nature. 2008;454(7203):436-444.

5. Chen J, Deng Q, Pan Y, et al. Prognostic value of neutrophil-tolymphocyte ratio in breast cancer. FEBS Open Bio. 2015;5:502-507.

6. $\mathrm{Hu} \mathrm{K}$, Lou L, Ye J, Zhang S. Prognostic role of the neutrophillymphocyte ratio in renal cell carcinoma: a meta-analysis. BMJ Open. 2015;5(4):e006404.

7. Xiao WK, Chen D, Li SQ, Fu SJ, Peng BG, Liang LJ. Prognostic significance of neutrophil-lymphocyte ratio in hepatocellular carcinoma: a meta-analysis. BMC Cancer. 2014;14:117.

8. Chen J, Hong D, Zhai Y, Shen P. Meta-analysis of associations between neutrophil-to-lymphocyte ratio and prognosis of gastric cancer. World J Surg Oncol. 2015;13:122.

9. Nagasaki T, Akiyoshi T, Fujimoto Y, et al. Prognostic impact of neutrophil-to-lymphocyte ratio in patients with advanced low rectal cancer treated with preoperative chemoradiotherapy. Dig Surg. 2015; 32(6):496-503.

10. Toiyama Y, Inoue Y, Kawamura M, et al. Elevated platelet count as predictor of recurrence in rectal cancer patients undergoing preoperative chemoradiotherapy followed by surgery. Int Surg. 2015;100(2): 199-207.

11. Wells GA, Shea B, Connell DO, et al. The Newcastle-Ottawa Scale (NOS) for assessing the quality of nonrandomised studies in metaanalyse. Available from: http://www.ohri.ca/programs/clinical_ epidemiology/oxford.asp. Accessed August 8, 2014.

12. Parmar MK, Torri V, Stewart L. Extracting summary statistics to perform meta-analyses of the published literature for survival endpoints. Stat Med. 1998;17(24):2815-2834.

13. Cochran WG. The combination of estimates from different experiments Biometrics. 1954;10:101-129.

14. Higgins JP, Thompson SG, Deeks JJ, Altman DG. Measuring inconsistency in meta-analyses. BMJ. 2003;327(7414):557-560.

15. Mantel N, Haenszel W. Statistical aspects of the analysis of data from retrospective studies of disease. J Natl Cancer Inst. 1959;22(4):719-748.

16. DerSimonian R, Laird N. Meta-analysis in clinical trials. Control Clin Trials. 1986;7(3):177-188.

17. Egger M, Davey Smith G, Schneider M, Minder C. Bias in meta-analysis detected by a simple, graphical test. BMJ. 1997;315(7109):629-634.

18. Carruthers R, Tho LM, Brown J, Kakumanu S, McCartney E, McDonald AC. Systemic inflammatory response is a predictor of outcome in patients undergoing preoperative chemoradiation for locally advanced rectal cancer. Colorectal Dis. 2012;14(10):e701-e707.

19. Liu H, Liu G, Bao Q, et al. The baseline ratio of neutrophils to lymphocytes is associated with patient prognosis in rectal carcinoma. J Gastrointest Cancer. 2010;41(2):116-120.

20. Harji D, Griffiths B, Sarveswaran J, Evans M, Koshy A, Sagar P. Neutrophil-lymphocyte ratio as a predictor of survival in locally recurrent rectal cancer. Colorectal Dis. 2012;14:10.

21. Kim IY, You SH, Kim YW. Neutrophil-lymphocyte ratio predicts pathologic tumor response and survival after preoperative chemoradiation for rectal cancer. BMC Surg. 2014;14:94.

22. Grivennikov SI, Greten FR, Karin M. Immunity, inflammation, and cancer. Cell. 2010;140(6):883-899.

23. Hanahan D, Weinberg RA. Hallmarks of cancer: the next generation. Cell. 2011;144(5):646-674.

24. Grivennikov SI, Karin M. Dangerous liaisons: STAT3 and NF-kappaB collaboration and crosstalk in cancer. Cytokine Growth Factor Rev. 2010;21(1):11-19.
25. Kishimoto T. Interleukin-6: from basic science to medicine -40 years in immunology. Ann Rev Immunol. 2005;23:1-21.

26. Lippitz BE. Cytokine patterns in patients with cancer: a systematic review. Lancet Oncol. 2013;14(6):e218-e228.

27. Popivanova BK, Kitamura K, Wu Y, et al. Blocking TNF-alpha in mice reduces colorectal carcinogenesis associated with chronic colitis. J Clin Invest. 2008;118(2):560-570.

28. Bollrath J, Phesse TJ, von Burstin VA, et al. gp130-mediated Stat3 activation in enterocytes regulates cell survival and cell-cycle progression during colitis-associated tumorigenesis. Cancer Cell. 2009; 15(2):91-102.

29. Becker C, Fantini MC, Schramm C, et al. TGF-beta suppresses tumor progression in colon cancer by inhibition of IL-6 trans-signaling. Immunity. 2004;21(4):491-501.

30. Grivennikov S, Karin E, Terzic J, et al. IL-6 and Stat3 are required for survival of intestinal epithelial cells and development of colitisassociated cancer. Cancer Cell. 2009;15(2):103-113.

31. Fridman WH, Pages F, Sautes-Fridman C, Galon J. The immune contexture in human tumours: impact on clinical outcome. Nat Rev Cancer. 2012;12(4):298-306.

32. Corthay A. Does the immune system naturally protect against cancer? Front Immunol. 2014;5:197.

33. Kawai O, Ishii G, Kubota K, et al. Predominant infiltration of macrophages and $\mathrm{CD} 8(+) \mathrm{T}$ cells in cancer nests is a significant predictor of survival in stage IV nonsmall cell lung cancer. Cancer. 2008; 113(6):1387-1395.

34. Ogino S, Nosho K, Irahara N, et al. Lymphocytic reaction to colorectal cancer is associated with longer survival, independent of lymph node count, microsatellite instability, and $\mathrm{CpG}$ island methylator phenotype. Clin Cancer Res. 2009;15(20):6412-6420.

35. Waldner M, Schimanski CC, Neurath MF. Colon cancer and the immune system: the role of tumor invading T cells. World J Gastroenterol. 2006;12(45):7233-7238.

36. Ostrand-Rosenberg S, Sinha P. Myeloid-derived suppressor cells: linking inflammation and cancer. J Immunol. 2009;182(8):4499-4506.

37. Mougiakakos D, Choudhury A, Lladser A, Kiessling R, Johansson CC. Regulatory T cells in cancer. Adv Cancer Res. 2010;107:57-117.

38. Hung HY, Chen JS, Yeh CY, et al. Effect of preoperative neutrophillymphocyte ratio on the surgical outcomes of stage II colon cancer patients who do not receive adjuvant chemotherapy. Int J Colorectal Dis. 2011;26(8):1059-1065.

39. Proctor MJ, Talwar D, Balmar SM, et al. The relationship between the presence and site of cancer, an inflammation-based prognostic score and biochemical parameters. Initial results of the Glasgow Inflammation Outcome Study. Br J Cancer. 2010;103(6):870-876.

40. Toiyama Y, Inoue Y, Saigusa S, et al. C-reactive protein as predictor of recurrence in patients with rectal cancer undergoing chemoradiotherapy followed by surgery. Anticancer Res. 2013;33(11):5065-5074.

41. Roxburgh CS, Salmond JM, Horgan PG, Oien KA, McMillan DC. The relationship between the local and systemic inflammatory responses and survival in patients undergoing curative surgery for colon and rectal cancers. J Gastrointest Surg. 2009;13(11):2011-2018; discussion 2018-2019.

42. Powell AG, Wallace R, McKee RF, et al. The relationship between tumour site, clinicopathological characteristics and cancer-specific survival in patients undergoing surgery for colorectal cancer. Colorectal Dis. 2012;14(12):1493-1499.

43. Kwon HC, Kim SH, Oh SY, et al. Clinical significance of preoperative neutrophil-lymphocyte versus platelet-lymphocyte ratio in patients with operable colorectal cancer. Biomarkers. 2012;17(3):216-222.

44. Azab B, Jaglall N, Atallah JP, et al. Neutrophil-lymphocyte ratio as a predictor of adverse outcomes of acute pancreatitis. Pancreatology. 2011; 11(4):445-452.

45. Park JJ, Jang HJ, Oh IY, et al. Prognostic value of neutrophil to lymphocyte ratio in patients presenting with ST-elevation myocardial infarction undergoing primary percutaneous coronary intervention. Am J Cardiol. 2013;111(5):636-642. 


\section{Publish your work in this journal}

OncoTargets and Therapy is an international, peer-reviewed, open access journal focusing on the pathological basis of all cancers, potential targets for therapy and treatment protocols employed to improve the management of cancer patients. The journal also focuses on the impact of management programs and new therapeutic agents and protocols on

patient perspectives such as quality of life, adherence and satisfaction. The manuscript management system is completely online and includes a very quick and fair peer-review system, which is all easy to use. Visit http://www.dovepress.com/testimonials.php to read real quotes from published authors.

Submit your manuscript here: http://www.dovepress.com/oncotargets-and-therapy-journal 